\title{
MODELOS DE MIGRACIÓN DEL PLOMO DE DOS ENVASES CELULÓSICOS EN CONTACTO CON SIMULANTE DE ALIMENTOS
}

\author{
Gerson Martín Cabrejos Hurtado*a, Walter Francisco Salas Valerio ${ }^{\text {a }}$
}

\begin{abstract}
RESUMEN
El objetivo de la investigación fue estimar modelos matemáticos para la migración del plomo $(\mu \mathrm{g} / \mathrm{l})$ desde una matriz celulósica hacia un simulante de alimentos de $\mathrm{pH}$ neutro, tomando dos variables independientes: la migración total $\left(\mathrm{mg} / \mathrm{dm}^{2}\right)$ y el área de contacto $\left(\mathrm{dm}^{2}\right)$, utilizando la regresión lineal múltiple. Las muestras evaluadas fueron dos envases celulósicos destinados al contacto con alimentos, siendo la procedencia de la celulosa de Chile y Brasil. Los ensayos de migración total y específica se realizaron con las metodologías de la Resolución Nº12 del MERCOSUR (1995) y el método 3111 B de la APHA (2005), respectivamente, bajo condiciones de contacto prolongado y a una temperatura de $50^{\circ} \mathrm{C}$. Los resultados de esta investigación demuestran que sólo el modelo para la celulosa de Brasil (Y $=-55,864-0,304 \mathrm{X} 1+9,173 \mathrm{X} 2$ ) resultó adecuado estadísticamente para realizar pronósticos, siendo su coeficiente de correlación (R2) de 0,899. Del análisis estadístico descriptivo, la variable migración específica del plomo con respecto a las variables migración total y área presentaron relación lineal, según el estadístico de Pearson $(0,854 / 0,944)$. El modelo para la celulosa de Chile ( $\mathrm{Y}=0,113+1,386 \mathrm{X} 1-0,085 \mathrm{X} 2)$ obtuvo un coeficiente de correlación (R2) de 0,615 y una moderada relación lineal entre las variables según el estadístico de Pearson $(0,784 / 0,538)$, siendo el modelo no adecuado para realizar pronósticos.
\end{abstract}

Palabras clave: envases celulósicos, migración total, migración específica, plomo.

\section{MODELS OF LEAD MIGRATION OF TWO CELLULOSE CONTAINERS IN CONTACT WITH FOOD SIMULANT}

\begin{abstract}
SUMMARY
The objective of the research was to estimate mathematical models for lead migration $(\mu \mathrm{g} / \mathrm{l})$ from a cellulose matrix using a neutral $\mathrm{pH}$ food simulant, taking two independent variables: total migration $\left(\mathrm{mg} / \mathrm{dm}^{2}\right)$ and the contact area $\left(\mathrm{dm}^{2}\right)$, using multiple linear regression. The evaluated samples were two cellulose containers destined to the contact with food, being

\footnotetext{
a Facultad de Industrias Alimentarias de la Universidad Nacional Agraria La Molina, Av. La Molina S/N, Lima, Perú.

*gerson.cabrejosh@ciplima.org.pe
} 
the origin of the cellulose from Chile and Brazil. Total and specific migration tests were carried out with the methodologies of Resolution No. 12 of MERCOSUR (1995) and method 3111 B of APHA (2005) respectively, under conditions of prolonged contact and at a temperature of $50{ }^{\circ} \mathrm{C}$. The results of this research show that only the model for Brazilian pulp $(\mathrm{Y}=-55,864-0,304 \mathrm{X} 1+9,173 \mathrm{X} 2)$ was statistically adequate to make forecasts, with its correlation coefficient (R2) of 0,899. From the descriptive statistical analysis, the variable specific migration of lead with respect to the variables total migration and area presented a linear relationship, according to the Pearson $(0,854 / 0,944)$ statistic. The model for Chilean pulp $(\mathrm{Y}=0,113+1,386 \mathrm{X} 1-0,085 \mathrm{X} 2)$ obtained a correlation coefficient (R2) of 0,615 and a moderate linear relationship between the variables according to the Pearson $(0,784 / 0,538)$ statistic, being the model not suitable for forecasting.

Key words: cellulose containers, total migration, specific migration, lead.

\section{INTRODUCCIÓN}

Siendo la celulosa un polímero natural de amplio uso en la industria, la transformación en envases para contacto directo con alimentos no está libre de la exposición al plomo, por ello es necesario un control durante la producción y distribución. Con el creciente uso de fibras celulósicas provenientes del material reciclado en la fabricación de envases en contacto con alimentos, existe la preocupación por el riesgo de migración de elementos tóxicos para los alimentos ${ }^{1,2,3,4,5}$. El material reciclado varía en el origen y puede incluir papeles-cartones conteniendo: tintas de impresión, adhesivos, ceras, colorantes, entre otros aditivos ${ }^{1,4}$. Los contaminantes que han sido encontrados frecuentemente en materiales celulósicos reciclados incluyen dioxinas y furanos, ftalatos, hidrocarburos policíclicos aromáticos y elementos potencialmente tóxicos como el plomo $^{3}$. La presencia de trazas de elementos potencialmente tóxicos en envases celulósicos para alimentos es un problema que está relacionado con la calidad final de los alimentos comercializados, pues estos elementos pueden migrar para el alimento en cantidades superiores a los límites de migración total o específica, representando un riesgo para la salud. Diversos investigadores evidenciaron plomo en la composición de envases celulósicos para alimentos bajo diferentes técnicas analíticas: ICP-MS $(0,1-6,6 \mathrm{mg} / \mathrm{kg})^{6}$; SS-GF-AAS $(0,14-6,60 \mathrm{mg} / \mathrm{kg})^{7}$; FAAS $(0,75-9,84 \mathrm{mg} / \mathrm{kg})^{8}$. De los estudios realizados por los autores se evidencia que la celulosa utilizada como material en contacto con alimentos presenta cantidades relevantes de plomo, por ello recomiendan realizar monitoreos del plomo como control de la inocuidad de los envases celulósicos en contacto con alimentos ${ }^{6}$.

Siendo los ensayos de migración específica no comerciales para el uso de las industrias, además, presentan factores particulares como: simulantes, técnica espectrométrica, entre otras; hacen que pocas o ninguna industria los realice. Por estos motivos investigadores proponen modelos matemáticos que reemplacen los ensayos de migración bajo condiciones predeterminadas, por ejemplo: en el modelamiento de la migración de aditivos de acrilonitrilo butadieno estireno para el agua como simulante ${ }^{9}$; en la migración del monómero de cloruro de vinilo de botellas de PVC para alimentos"; en el modelo cinético a través de un proceso 
de sorción/desorción para migrantes de bajo peso molecular capaces de difundirse a través del polímero ${ }^{10}$; otros autores desarrollaron la teoría del volumen libre, incluyendo hasta las moléculas grandes como los plastificantes ${ }^{9}$. Existe numerosa investigación sobre modelos de migración en materiales poliméricos sintéticos comparado con polímeros naturales.

El autor, en su investigación, propone un modelo de desorción del cadmio $(\mu \mathrm{g} / \mathrm{l})$ a partir de tres variables independientes: concentración inicial $(\mathrm{mg} / \mathrm{kg})$, temperatura $\left({ }^{\circ} \mathrm{C}\right)$ y tiempo (min.), desde un material celulósico hacia agua como simulante. Su modelo obtuvo un coeficiente de correlación $\left(\mathrm{R}^{2}\right)$ de 0,92 y de 0,91 para los resultados de validación, recomendando este para estimar pronósticos ${ }^{2}$.

Además de los trabajos sobre modelos para migración, pocos datos prácticos fueron publicados para evaluar esos modelos, siendo estos concentrados en sustancias que migran de forma independiente, aproximándose a los modelos ficknianos, como la migración de monómeros para simulantes acuosos. El desarrollo de modelos matemáticos para la migración tiene muchas ventajas, sin embargo, todavía no pueden sustituir a los ensayos de migración en la evaluación del potencial de contaminación del alimento por sustancias químicas procedentes del envase 9 .

En ese contexto el presente trabajo de investigación tuvo como objetivo estimar modelos matemáticos para dos muestras de celulosa, siendo su procedencia de Brasil y Chile. La variable dependiente fue la migración específica del plomo y las variables independientes: la migración total $\left(\mathrm{mg} / \mathrm{dm}^{2}\right)$ y área de contacto $\left(\mathrm{dm}^{2}\right)$, bajo las condiciones de tiempo prolongado y una temperatura de $50^{\circ} \mathrm{C}$. Esta investigación beneficiará a las industrias fabricantes de envases celulósicos y a los consumidores en general, permitiendo utilizar las metodologías y modelos matemáticos propuestos, además de conocer los riesgos asociados a la contaminación con el plomo por materiales en contacto con alimentos.

\section{PARTE EXPERIMENTAL}

\section{Acondicionamiento de la muestra}

Las muestras de envases celulósicos, de origen Brasil y Chile, fueron proveídas de las industrias productoras en Lima-Perú, entregados en bolsas de polietileno y almacenados en un ambiente cerrado alejado de otros compuestos químicos utilizados en el laboratorio de Biotecnología de la FIAL-UNALM, según el procedimiento establecido por la referencia ${ }^{6}$. Las muestras presentaron estructuras de tres y dos pliegos de celulosa equivalente a gramajes de 210 y 170 gramos $/ \mathrm{m}^{2}$, respectivamente.

En la elección de las características del simulante A, se utilizó agua grado reactivo para análisis clase II que lo establece la referencia ${ }^{11}$, por ser la más apropiada para realizar análisis por presentar menos interferencias durante el análisis espectrométrico de absorción atómica. 


\section{Diseño experimental}

Se analizaron dos muestras de envases celulósicos (Chile y Brasil), se redujo el número de repeticiones de cuatro ${ }^{12}$ a tres ${ }^{6}$ por incrementarse el área de contacto (mayor de $6 \mathrm{dm}^{2}$ ), siendo también propuesta esa cantidad por la referencia. En cada modelo de regresión se consideraron las áreas obtenidas por los ensayos de migración específica y total, siendo nueve el número total de áreas por modelo.

\section{Migración total}

Las muestras se cortaron en tamaños de acorde al equipo de sujeción, siendo el área aproximada por ensayo de $6 \mathrm{dm}^{2}$, colocándolas en contacto con el simulante A. Se acondicionaron los equipos de sujeción en una estufa a $50{ }^{\circ} \mathrm{C}$ por 24 horas, luego se recuperó el extracto de simulante en un vaso precipitado para evaporarlo hasta $5 \mathrm{ml}$, aproximadamente. Finalmente, se terminó de evaporar el extracto en una mufla a $105^{\circ} \mathrm{C}$ hasta peso constante, siendo el residuo obtenido almacenado en un desecador hasta enfriarse y pesarse ${ }^{12}$. Los resultados de migración total se calcularon: $\mathrm{MT}=($ Peso del residuo - Peso del blanco) / Área de contacto.

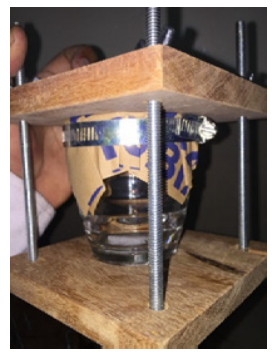

Figura 1. Dispositivo de fijación, simulante A y muestra de envase celulósico.

\section{Migración específica del plomo}

Se realizó el ensayo de migración total para obtener el extracto del contacto entre el simulante y las muestras celulósicas ${ }^{12}$, luego se procedió con la determinación del plomo en la muestra líquida según el método de análisis propuesto en referencia ${ }^{11}$. Se transfirieron $250 \mathrm{ml} \mathrm{de} \mathrm{cada}$ muestra por triplicado a un vaso precipitado, se adicionaron $5 \mathrm{ml} \mathrm{de} \mathrm{HNO}_{3}$ y se calentaron hasta desprendimiento de vapores rojizos. Luego se enfriaron a temperatura ambiente y se disgregaron las sales con 2,5 $\mathrm{ml}$ de $\mathrm{HCl}$. Finalmente, se transfirieron las muestras digestadas a un matraz de $25 \mathrm{ml}$ y se aforaron con agua destilada ${ }^{11}$. Se determinaron las concentraciones de plomo con el espectrómetro de absorción atómica novAA 300 (Analytik Jena, Alemania), los resultados de migración específica se calcularon: $\mathrm{ME}=$ (Concentración de plomo en la muestra - Concentración de plomo en el blanco) * Factor de dilución.

\section{Análisis estadístico}

Para el análisis estadístico descriptivo de las variables (diagrama de cajas, matriz de dispersión, histogramas de frecuencia, coeficientes de Pearson y Spearman) y el modelo matemático (coeficientes de los modelos, bondad de ajuste, significancia de los coeficientes 
y el coeficiente de correlación) se utilizó el software SPSS 23 (IBM, USA). Para el análisis de la normalidad de los residuos se utilizó el software Minitab 17 (Minitab Inc., USA).

\section{RESULTADOS Y DISCUSIÓN}

\section{Migración total y específica - muestra Chile}

De la tabla 1 se puede apreciar que los resultados obtenidos para la muestra que proviene de Chile, para la migración total fueron entre $0,84-6,89 \mathrm{mg} / \mathrm{dm}^{2}$, dando una media de 4,87 $\mathrm{mg} / \mathrm{dm}^{2}$. Del análisis descriptivo del diagrama de cajas en la figura 2 se observa asimetría a la izquierda de los valores normalizados (Z), una baja variabilidad de los resultados y dos valores atípicos $(0,84 ; 1,60)$. La variabilidad se debe al rango $\left(6,05 \mathrm{mg} / \mathrm{dm}^{2}\right)$ obtenido de los valores originales, siendo la repetición $\mathrm{CH} 1,2\left(0,84 \mathrm{mg} / \mathrm{dm}^{2}\right)$ muy por debajo de los valores conseguidos, por haberse obtenido de un área inferior $\left(4,86 \mathrm{dm}^{2}\right)$. De los resultados obtenidos de migración específica del plomo se encontraron valores entre 2,00-12,90 $\mu \mathrm{g} / \mathrm{l}$, siendo la media $(6,30 \mu \mathrm{g} / \mathrm{l})$. Del análisis descriptivo del diagrama de cajas en la figura $2 \mathrm{se}$ observa asimetría a la derecha de los valores normalizados (Z), una elevada variabilidad de los resultados y ningún valor atípico. Para la variable área se obtuvieron valores entre 4,86-7,95 $\mathrm{dm}^{2}$ dando una media de $6,53 \mathrm{dm}^{2}$, del análisis descriptivo los valores presentaron simetría y alta variabilidad.

Tabla 1. Resultados de los ensayos de migración específica y total de las muestras de Chile.

\begin{tabular}{lccc}
\hline Repetición & $\begin{array}{c}\text { Migración } \\
\text { Específica }(\boldsymbol{\mu g} / \mathbf{l})\end{array}$ & $\begin{array}{c}\text { Migración Total } \\
\left(\mathbf{m g} / \mathbf{d m}^{2}\right)\end{array}$ & Área $\left(\mathbf{d m}^{2}\right)$ \\
\hline CH1,1 & $\mathbf{3 , 3 0}$ & $\mathbf{4 , 7 5}$ & $\mathbf{7 , 9 5}$ \\
CH1,2 & $\mathbf{2 , 0 0}$ & $\mathbf{0 , 8 4}$ & $\mathbf{4 , 8 6}$ \\
CH1,3 & $\mathbf{3 , 2 0}$ & $\mathbf{1 , 6 0}$ & $\mathbf{5 , 7 4}$ \\
CH2,1 & $\mathbf{1 0 , 1 0}$ & $\mathbf{6 , 8 9}$ & $\mathbf{6 , 6 3}$ \\
CH2,2 & $\mathbf{5 , 3 0}$ & $\mathbf{6 , 0 2}$ & $\mathbf{6 , 6 3}$ \\
CH2,3 & $\mathbf{2 , 8 0}$ & $\mathbf{4 , 5 3}$ & $\mathbf{5 , 7 4}$ \\
CH3,1 & $\mathbf{8 , 3 0}$ & $\mathbf{6 , 1 2}$ & $\mathbf{6 , 1 8}$ \\
CH3,2 & $\mathbf{8 , 9 0}$ & $\mathbf{6 , 4 2}$ & 7,51 \\
CH3,3 & $\mathbf{1 2 , 9 0}$ & $\mathbf{6 , 6 6}$ & $\mathbf{7 , 5 1}$ \\
\hline
\end{tabular}

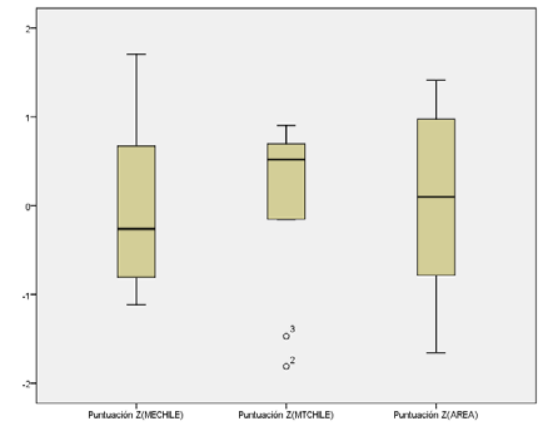

Figura 2. Gráfico de cajas de los valores $\mathrm{Z}$ para las variables (migración específica, migración total y área) en estudio en la muestra de Chile. 
En la figura 3 se presentan los diagramas de dispersión donde se visualiza la moderada relación lineal entre las variables de migración específica y migración total, esto se confirma con el estadístico de Pearson $(0,784)$, mientras que para las variables migración específica y área presentan moderada relación lineal lo cual se visualiza en los diagramas de dispersión y se verifica con el estadístico de Pearson $(0,538)$. Las variables de migración total y área también presentaron moderada relación lineal según los diagramas de dispersión y estadístico de Pearson $(0,700)$.

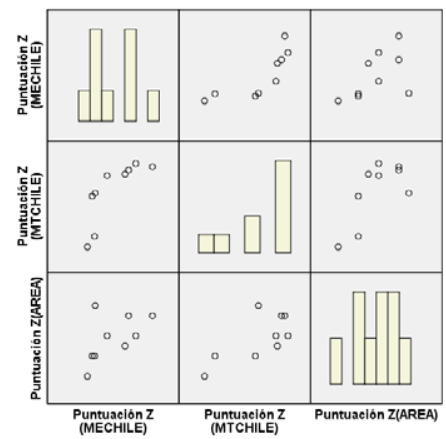

Figura 3. Matriz de dispersión de puntos para los valores $\mathrm{Z}$ de las variables (migración específica, migración total y área) en estudio en la muestra de Chile.

\section{Modelo de migración del plomo - muestra Chile}

Del análisis de regresión lineal para las variables en estudio se obtuvo el modelo: $\mathrm{Y}=0,113+$ 1,386X1 - 0,085X2 y un $\mathrm{R}^{2}=0,615$; $\mathrm{R} 2 \mathrm{ajustado}=0,487$; $\mathrm{Se}=2,76344$; el modelo se considera regular por no encontrarse el coeficiente de correlación tan próximo a la unidad, ver figura 4.

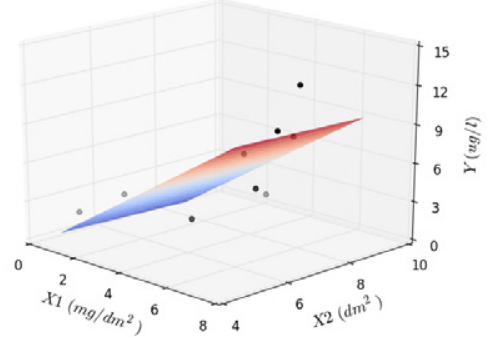

Figura 4. Modelo de migración del plomo $(\mathrm{Y})$ con respecto al área (X2) y migración total (X1) de las muestras de Chile.

Para la prueba de Anderson Darling para verificar la normalidad de los residuos del modelo, para un $\alpha=0,05$ se obtuvo un $\mathrm{p}$ valor de 0,183 , entonces no se rechaza la hipótesis nula o los residuos tienen distribución normal. Las medidas de influencia en el modelo se estimaron con el estadístico Cook's Distance y se obtuvo un dato influyente, sin embargo, se considera para la presente investigación. La correlación de los residuos del modelo se obtuvo mediante el estadístico de Durbin-Watson y su valor fue de 1,154 concluyendo que los residuos se 
encuentran incorrelacionados. Para la multicolinealidad de las variables del modelo se obtuvo: $1<\mathrm{VIF}<5$, concluyendo que existe moderada correlación de multicolinealidad. Para determinar la significancia del modelo se realizaron las pruebas de hipótesis para cada coeficiente de las variables (Ho: $\beta 1=0 ; \mathrm{H} 1: \beta 1 \neq 0 ; \alpha=0,05$ ), se obtuvieron $\mathrm{p}$ valores de 0,065 y 0,952 concluyendo el no rechazo de la Ho, sin embargo, para esta investigación se consideraron los coeficientes del modelo como significativos. Finalmente, para determinar si el modelo es adecuado para realizar pronósticos, se obtuvo la sumatoria de cuadrados totales (SCT) del ANOVA siendo el valor de 119,109, y la sumatoria de los residuos eliminados al cuadrado (PRESS) de 45,809. De los datos anteriores se obtuvo un $\mathrm{R}^{2}=0,615$, por ello se determina que el modelo no es adecuado para realizar pronósticos.

\section{Migración total y específica - muestra Brasil}

De la tabla 2 se puede apreciar que los resultados obtenidos para la migración total fueron entre 12,62-23,25 mg/dm², dando una media de $16,85 \mathrm{mg} / \mathrm{dm}^{2}$. Del análisis descriptivo del diagrama de cajas en la figura 5 se observa simetría de los valores normalizados $(Z)$, una elevada variabilidad de los resultados y ningún valor atípico. De los resultados obtenidos de migración específica del plomo se encontraron valores entre 3,70-17,10 $\mu \mathrm{g} / \mathrm{l}$, siendo la media $(9,71 \mu \mathrm{g} / \mathrm{l})$. Del análisis descriptivo del diagrama de cajas en la figura 5 se observa simetría de los valores normalizados $(Z)$, una elevada variabilidad de los resultados y ningún valor atípico. Para la variable área se obtuvieron valores entre 7,07-8,84 $\mathrm{dm}^{2}$ dando una media de $7,71 \mathrm{dm}^{2}$, del análisis descriptivo los valores presentaron asimetría a la derecha, alta variabilidad y un valor atípico.

Tabla 2. Resultados de los ensayos de migración específica y total de las muestras de Brasil.

\begin{tabular}{lccc}
\hline Repetición & $\begin{array}{c}\text { Migración } \\
\text { Específica }(\mu \mathrm{g} / \mathbf{l})\end{array}$ & $\begin{array}{c}\text { Migración Total } \\
\left(\mathbf{m g} / \mathbf{d m}^{2}\right)\end{array}$ & Área $\left(\mathbf{d m}^{2}\right)$ \\
\hline BR1,1 & $\mathbf{3 , 7 0}$ & $\mathbf{1 2 , 6 2}$ & $\mathbf{7 , 0 7}$ \\
BR1,2 & $\mathbf{9 , 3 0}$ & $\mathbf{1 4 , 8 5}$ & $\mathbf{7 , 5 1}$ \\
BR1,3 & $\mathbf{1 3 , 2 0}$ & $\mathbf{1 8 , 6 8}$ & $\mathbf{7 , 9 5}$ \\
BR2,1 & $\mathbf{6 , 0 0}$ & $\mathbf{1 0 , 9 5}$ & $\mathbf{7 , 0 7}$ \\
BR2,2 & $\mathbf{5 , 3 0}$ & $\mathbf{1 7 , 6 3}$ & $\mathbf{7 , 5 1}$ \\
BR2,3 & $\mathbf{1 4 , 8 0}$ & $\mathbf{2 1 , 1 9}$ & $\mathbf{8 , 3 9}$ \\
BR3,1 & $\mathbf{8 , 5 0}$ & $\mathbf{1 4 , 5 8}$ & $\mathbf{7 , 5 1}$ \\
BR3,2 & $\mathbf{1 7 , 1 0}$ & $\mathbf{2 3 , 2 5}$ & $\mathbf{8 , 8 4}$ \\
BR3,3 & $\mathbf{9 , 5 0}$ & $\mathbf{1 7 , 8 6}$ & $\mathbf{7 , 5 1}$ \\
\hline
\end{tabular}

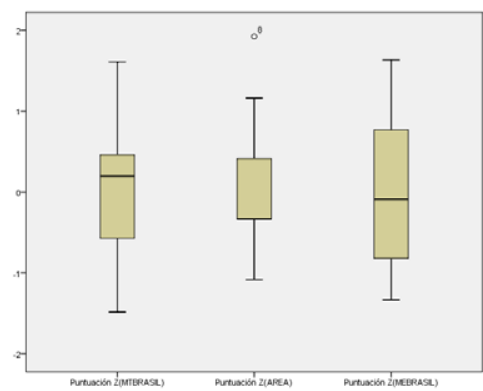

Figura 5. Gráfico de cajas de los valores $\mathrm{Z}$ para las variables (migración específica, migración total y área) en estudio en la muestra de Brasil. 
En la figura 6 se presentan los diagramas de dispersión donde se visualiza moderada relación lineal entre las variables de migración específica y migración total, esto se confirma con el estadístico de Pearson $(0,854)$, mientras que para las variables migración específica y área presentan relación lineal, lo cual se visualiza en los diagramas de dispersión y se verifica con el estadístico de Pearson $(0,944)$. Las variables de migración total y área también presentaron relación lineal según los diagramas de dispersión y estadístico de Pearson $(0,939)$.

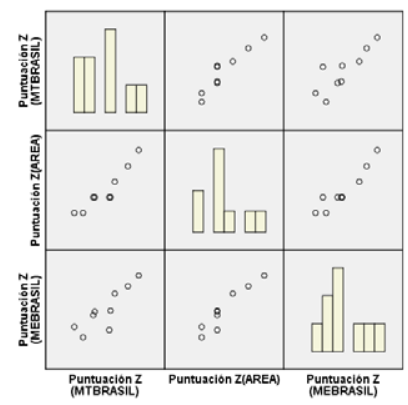

Figura 6. Matriz de dispersión de puntos para los valores $\mathrm{Z}$ de las variables (migración específica, migración total y área) en estudio en la muestra de Brasil.

\section{Modelo de migración del plomo - muestra Brasil}

Del análisis de regresión lineal para las variables en estudio se obtuvo el modelo: $\mathrm{Y}=-55,864$ $-0,304 X 1+9,173 X 2$ y un $R^{2}=0,899 ; R^{2} a j u s t a d o=0,865 ; S_{e}=1,65937 ;$ el modelo se considera apropiado por encontrarse el coeficiente de correlación próximo a la unidad, ver figura 7.

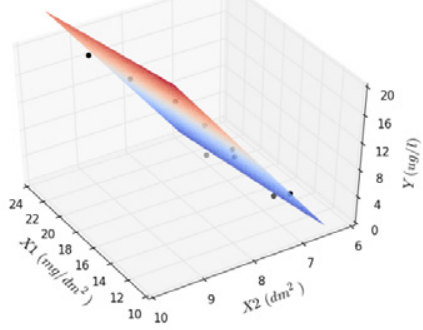

Figura 7. Modelo de migración del plomo (Y) con respecto al área (X2) y migración total (X1) de las muestras de Brasil.

Para la prueba de Anderson Darling para verificar la normalidad de los residuos del modelo, para un $\alpha=0,05$ se obtuvo un $\mathrm{p}$ valor de 0,856 , entonces no se rechaza la hipótesis nula o los residuos tienen distribución normal. Las medidas de influencia en el modelo se estimaron con el estadístico Cook's Distance y no se obtuvieron datos influyentes. La correlación de los residuos del modelo se obtuvo mediante el estadístico de Durbin-Watson y su valor fue de 1,916 concluyendo que los residuos se encuentran incorrelacionados. Para la multicolinealidad de las variables del modelo se obtuvo: $5<\mathrm{VIF}$, concluyendo que existe alta correlación de 
multicolinealidad, sin embargo para la presente investigación se continuó con los análisis de regresión. Para determinar la significancia del modelo se realizaron las pruebas de hipótesis para cada coeficiente de las variables (Ho: $\beta 1=0 ; H 1: \beta 1 \neq 0 ; \alpha=0,05$ ), se obtuvieron $p$ valores de 0,505 y 0,019 , concluyendo el no rechazo de la Ho para la variable X1 y el rechazo de la Ho para la variable X2, sin embargo para esta investigación se consideró el coeficiente de la variable X1 del modelo como significativo. Finalmente, para determinar si el modelo es adecuado para realizar pronósticos, se obtuvo la sumatoria de cuadrados totales (SCT) del ANOVA siendo el valor de 163,709 , y la sumatoria de los residuos eliminados al cuadrado (PRESS) de 16,514. De los datos anteriores se obtuvo un $\mathrm{R}^{2}=0,899$, por ello se determina que el modelo es adecuado para realizar predicciones.

Se utilizó el método estadístico de regresión lineal múltiple porque existe relación lineal entre las variables en estudio, siendo la transferencia de masa de la migración específica considerada parte de la migración total, además el área de contacto contribuye a un mejor ajuste del modelo. La naturaleza de la fase de contacto polímero/alimento o simulante influencia tanto al coeficiente de difusión como al coeficiente de partición ${ }^{13}$. En este aspecto, el autor clasificó el potencial de migración en tres tipos básicos, siendo nuestro caso de clase III o controlada por al alimento o simulante. De la clase III se puede observar una aproximada relación lineal entre la migración y el tiempo al final de la curva, lo cual refuerza nuestra propuesta del modelo lineal para tiempo prolongado. Otros autores realizaron ensayos sobre la transferencia del cadmio desde pulpa virgen de papel hacia un simulante acuoso neutro, sus resultados demostraron que para periodos de tiempo prolongado la temperatura no es un factor importante después de tres horas de contacto, obteniendo menos del $20 \%$ de migración del analito ${ }^{2}$. Entonces podemos asegurar que la temperatura en nuestro modelo no interfiere en los resultados, a pesar que se mantuvo constante durante los ensayos $\left(50{ }^{\circ} \mathrm{C}\right)$. Otro factor que soporta nuestro modelo lineal es el tiempo prolongado ( 24 horas), siendo que los autores demostraron que el mayor porcentaje de migración ocurre en las primeras tres horas de contacto ${ }^{14}$, por ello pasado este tiempo la transferencia de masa pasa de un modelo exponencial a uno lineal.

El modelo de migración del plomo de la muestra de Chile $(\mathrm{Y}=0,113+1,386 \mathrm{X} 1-0,085 \mathrm{X} 2$; $\left.\mathrm{R}^{2}=0,615\right)$ no resultó adecuado para realizar pronósticos porque los resultados obtenidos presentaron moderada relación lineal, lo cual no cumple con la teoría sobre la relación directa entre la migración específica y total. Esta relación se cumple para la migración del plomo porque la técnica gravimétrica para determinar la migración total solo separa los compuestos y/o elementos volátiles, además la técnica espectrométrica requiere del extracto obtenido de la migración total.

Los autores de la referencia estimaron un modelo cinético del cadmio en celulosa virgen a través de agua de $\mathrm{pH}$ neutro, obtuvieron un $\mathrm{R}^{2}=0,89$, concluyendo que su modelo permite realizar pronósticos. Entonces también podemos concluir que nuestro modelo de migración del plomo en la muestra de Brasil ( $\left.\mathrm{Y}=-55,864-0,304 \mathrm{X} 1+9,173 \mathrm{X} 2 ; \mathrm{R}^{2}=0,899\right)$ es adecuado para realizar pronósticos, el cual permite a los consumidores estimar las concentraciones del plomo bajo las mismas condiciones de ensayo, evitando realizar los ensayos de migración específica. 


\section{CONCLUSIONES}

Los modelos de migración del plomo de envases celulósicos en contacto con el simulante A obtenidos fueron: Chile $\left(\mathrm{Y}=0,113+1,386 \mathrm{X} 1-0,085 \mathrm{X} 2 ; \mathrm{R}^{2}=0,615 ; \mathrm{R}^{2}\right.$ ajustado=0,487; $\left.\mathrm{S}_{\mathrm{e}}=2,76344\right)$ y Brasil $\left(\mathrm{Y}=-55,864-0,304 \mathrm{X} 1+9,173 \mathrm{X} 2 ; \mathrm{R}^{2}=0,899 ; \mathrm{R} 2\right.$ ajustado=0,865; $\mathrm{S}_{\mathrm{e}}=1,65937$ ), siendo sólo el modelo de Brasil adecuado para realizar pronósticos por los fabricantes y consumidores, según el análisis descriptivo de las variables que determinaron relación lineal y el análisis de regresión lineal múltiple que determinó la significancia del modelo.

\section{AGRADECIMIENTOS}

Al profesor Walter Francisco Salas Valerio de la Facultad de Industrias Alimentarias de la UNALM y al equipo del Laboratorio de Biotecnología de la FIAL-UNALM por su apoyo durante la investigación.

\section{REFERENCIAS BIBLIOGRÁFICAS}

1. Binderup ML, Pedersen GA, Vinggaard AM, Rasmussen ES, Rosenquist H, Cederberg T. Toxicity testing and chemical analyses of recycled fibre-based paper for food contact. Food Addit Contam. 2002; 19:13-28.

2. García GC, Carbonell G, Tarazona JV. Modelling the absorption and desorption of cadmium on paper pulp using kinetic approaches. Chemosphere. 2003; 55:869-878.

3. Triantafyllou VI, Akrida-Demertzis PG. Migration studies from recycled paper packaging materials: development of an analytical method for rapid testing. Anal Chim Acta. 2002; 467:253-260.

4. Triantafyllou VI, Akrida-Demertzis PG. A study on the migration of organic pollutants from recycled paperboard packaging materials to solid food matrices. Food Chem. 2007; 101:1759-1768.

5. Skrzydlewska E, Balcerzak M, Vanhaecke F. Determination of chromium, cadmium and lead in food-packaging materials by axial inductively coupled plasma time-of-flight mass spectrometry. Anal Chim Acta. 2002; 479:191-202.

6. Castle L, Offen CP, Baxter MJ, Gilbert J. Migration studies from paper and board food packaging materials, 1. Compositional analysis. Food Addit Contam. 1997; 14(1):35-44.

7. De Sousa Medeiros P. Desenvolvimentos de métodos analíticos para determinacao de $\mathrm{As}, \mathrm{Cd}, \mathrm{Hg}, \mathrm{Cr}$ e $\mathrm{Pb}$ em embalagens celulósicas para alimentos por espectrometría de absorcao atómica e amostragem direta de solidos. [Dissertacao para obtencao do título de Mestre em Química]. Sao Paulo-Brasil: Universidade de Sao Paulo; 2009.

8. Cioban CG. Heavy metal distribution in different type of paper packaging. Annals of the University of Craiova. 2015; 20(56):97-100. 
9. Correa GE. Estudo do potencial de migracao de componentes de embalagens plasticas para produtos gordurosos a altas temperaturas. [Tese para obtencao do título de Mestre em Tecnologia de Alimentos]. Sao Paulo-Brasil: Universidade Estadual de Campinas; 1996.

10. Gilbert SG, Miltz J, Giancin JR. Transport considerations of potential migrants from food packaging materials. J Food Process Preserv.1980; 4:27-48.

11. APHA. Standard Methods for the Examination of Water and Wastewater, 3111 B: Direct Air-Acetylene Flame Method. 21th Edition. Washington-United States of America: American Public Health Association; 2005.

12. Resolución $\mathrm{N}^{\circ} 12$. Ensayo de migración total de envases y equipamientos celulósicos. Paraguay: Grupo Mercado Común del Sur; 1995.

13. Crosby NT. Food packaging materials: aspects of analysis and migration of contaminants. London-England: Applied Science; 1981.

14. García GC, Carbonell G, Tarazona JV. Binding of cadmium on raw paper pulp: relationship between temperature and sorption kinetics. Chemosphere. 2002; 49:533538. 